\title{
Des voies d'évolution pour les matériaux utilisés dans le matériel roulant SNCF
}

\author{
Patrick Lallet ${ }^{1}$ et Francis Cocheteux ${ }^{2}$ \\ ${ }^{1}$ Agence d'Essai Ferroviaire, 21 avenue S. Allende, 94407 Vitry-sur-Seine, France \\ 2 Direction du Matériel-Département Sécurité, Qualité et Gestion des risques, 15 rue Traversière, 75580 Paris, \\ France
}

\begin{abstract}
Résumé. La recherche de la performance a été, dès les débuts du chemin de fer, une forte motivation des techniciens et ingénieurs. Si les premiers matériels étaient essentiellement en bois, l'introduction de matériaux métalliques dans les structures a permis des progrès notables, notamment au regard de la résistance lors de collision. D'importants travaux, en collaboration avec les industriels, ont ainsi été entrepris afin de bien connaître les propriétés des matériaux utilisés sur nos trains, telle que la tenue en fatigue afin d'optimiser les structures tout en garantissant un haut niveau de sécurité. Plus tard, la recherche d'allègement des matériels a débouché sur l'aluminium et les matériaux composites permettant ainsi le transport d'un nombre plus important de voyageurs sans augmenter la longueur des quais ou le cadencement des circulations et cela via des concepts de matériels à deux niveaux. Plus récemment, les règlements et directives européennes, fixant l'ouverture des marchés, a conduit la SNCF à exprimer ses besoins de manière fonctionnelle et à changer ses modes de spécifications. Enfin, la prise en compte du développement durable est à présent une des préoccupations majeures de la SNCF dont les matériaux sont en partie contributifs.
\end{abstract}

\section{INTRODUCTION}

L'évolution des règles de conception des véhicules ferroviaires, est bâtie sur un besoin de standardisation et sur la satisfaction de critères aussi divers que le confort dynamique, la durée de vie, la légèreté et la sécurité en service. Les véhicules sont soumis à une multitude de sollicitations auxquels ils doivent résister pendant leur vie opérationnelle tout en étant inter opérables. Les réseaux se sont donc attachés à dimensionner les structures vis à vis des sollicitations d'épreuves déterminées à partir de conditions extrêmes du service. Du fait qu'il est indispensable que les véhicules continuent à assurer un service normal après ces sollicitations, les structures doivent rester en tous points dans le domaine d'élasticité.

\section{L'OPTIMISATION DE LA PERFORMANCE}

Quelques exemples permettent d'illustrer les évolutions réalisées et d'envisager l'avenir :

\section{La caisse}

A l'origine du ferroviaire, les premiers véhicules étaient basés sur les architectures de carrosse avec un châssis métallique riveté et une superstructure en bois. Ils consistaient en des véhicules isolés reliés entre eux par des attelages de traction et des tampons de chocs et étaient attelés à un engin moteur pour former un train. Puis, pour prendre en compte la sécurité des occupants, mise en cause à la suite d'accidents, des voitures de lignes basées sur la même conception ont été reprises peu à peu pour renforcer la superstructure en bois par adjonction d'un tôlage métallique [1].

Les véhicules ont ensuite évolué vers une structure tubulaire, construite entièrement en acier et/ou en acier inox soudée par points, puis soudée de manière continue. En effet, Il était devenu évident que la juxtaposition d'un châssis robuste et d'une caisse n'offrait pas la sécurité optimale en cas de choc. Après accident, le châssis ne présentait que peu de dommages, alors que la caisse «voyageurs» était complètement détruite. De plus, lors des collisions ou des déraillements de structures rivetées, on constatait l'arrachement des tôles et leur refoulement après striction au droit des lignes de rivets (avec éclatement de la caisse et télescopage), à la manière d'une étoffe qu'on fait céder sur ses coutures. Les soudures continues ont ainsi apporté un caractère plus sécuritaire de part des points d'ancrage pratiquement invulnérables aux bords des tôles qu'ils relient soit entre elles, soit aux membrures principales de châssis et de caisse.

A la suite de ces accidents historiques, les bureaux d'études étaient totalement polarisés sur le problème si préoccupant de la Sécurité. Ils n'entrevoyaient pas encore les pleines possibilités d'allégement offertes par l'optimisation des conceptions de véhicules en acier et ensuite par l'utilisation d'aciers ou d'alliages d'aluminium performants. Ce n'est donc qu'à partir des années 80 que des véhicules à un ou deux niveaux ont été conçus en structures tubulaires faites de profilés aluminium de types simple ou double peau, extrudés, soudés... ou avec des aciers à haute limite d'élasticité.

A l'avenir, il faudra continuer à alléger. Développer des solutions multi-matériaux alliant par exemple l'acier, l'aluminium et les matériaux composites [2].

\section{Les pantographes}

Pour les matériels moteurs à traction électrique, le pantographe représente l'organe complexe par excellence et les propriétés des matériaux demeurent essentielles (résistance, usure, tribologie, conductivité...). 


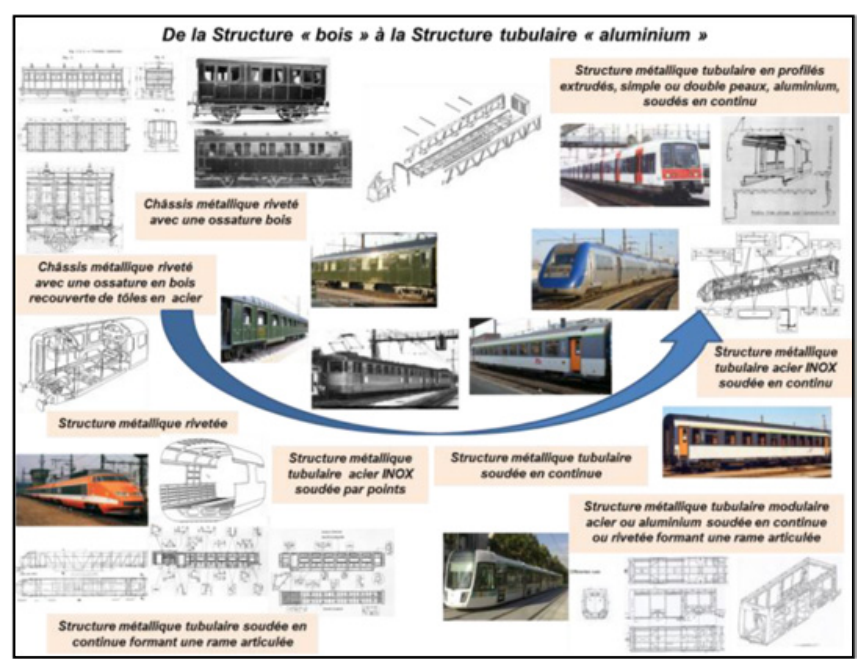

Figure 1. De la structure bois à la structure aluminium.

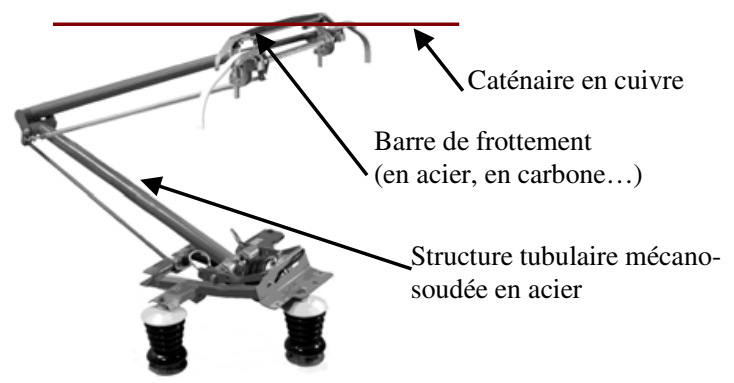

Figure 2. Pantographe AMDE.

Les exigences du contact panto-caténaire impliquent nécessairement la recherche de compromis sur les propriétés des matériaux : Pour les applications $25 \mathrm{kV}$, le matériau de frottement est exclusivement le carbone amorphe, dit «pur» (sans additif métallique) ou imprégné de cuivre. Les faibles niveaux de courant permettent d'obtenir de grandes durées de vie des caténaires (50 à 75 ans) et des bandes de frottement (jusqu'à $80000 \mathrm{~km}$ sur $\mathrm{TGV}, 200000 \mathrm{~km}$ pour des automotrices.

Pour les applications $1,5 \mathrm{kV}$, le cuivre et de l'acier sont les matériaux les plus utilisés du fait des plus forts courants $(2500$ à $5000 \mathrm{~A})$. Les durées de vie sont courtes: de 2 à 10 ans pour les portions de caténaires très sollicitées, de 8000 à $12000 \mathrm{~km}$ pour les archets TGV, de 5000 à $15000 \mathrm{~km}$ pour ceux des locomotives, parfois l'archet ne tient qu'un aller retour en cas de givre sur la caténaire.

Cette solution nécessite par ailleurs un graissage régulier des fils de contact et des archets.

La France est un des derniers pays d'Europe, avec l'Espagne, à utiliser des bandes de frottement en métal mais la problématique française est certainement une des plus difficile pour plusieurs raisons:

- Le cuivre pur utilisé pour les fils de contact s'échauffe rapidement en situation de captage à l'arrêt (risque de rupture)

- Les courants de traction peuvent être maintenus sur de très longues durées du fait des reliefs rencontrés (rampes de Modane, Blaisy, Souillac...). Les bandes

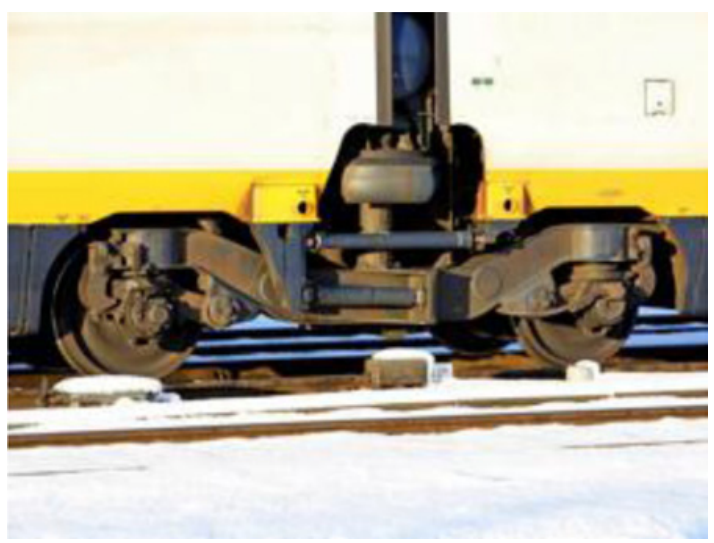

Figure 3. Châssis de bogie TGV.

de frottement s'échauffent se qui entraîne parfois leur dégradation.

L'avenir consistera donc principalement à résoudre ces difficultés.

\section{Les bogies}

Sous une caisse ou aux inter-caisses dans le cas du TGV, on trouve les bogies.

La masse d'un châssis de bogie est de l'ordre d'une à deux tonnes.

Hormis le cas de certains bogies Fret, les châssis de bogie sont en acier mécano-soudé. L'acier utilisé est un acier relativement standard (acier pour les structures - non allié), avec un critère de résilience à froid (afin d'éviter une rupture brutale en cas de choc par grand froid).

Compte tenu que les châssis de bogie sont mécano soudés, d'importants travaux ont été entrepris sur la connaissance des matériaux utilisés et leurs procédés d'assemblage.

Dans le cas de structure soudée, les fissures de fatigue peuvent s'amorcer en racine ou en pied de cordon. Ce sont les sites privilégiés d'amorçage car on y trouve de fortes concentrations de contrainte. Cette situation est due à la géométrie de la structure et du cordon (effet d'entaille) et aux facteurs inhérents au procédé de soudage tels que les irrégularités géométriques du cordon.

A ces facteurs géométriques, qui affectent la tenue en fatigue, viennent s'ajouter des facteurs métallurgiques, comme les inclusions ou encore le niveau des contraintes résiduelles. Le phénomène de fatigue est donc localisé dans une zone où les propriétés du matériau sont difficiles à évaluer et les géométries complexes et accidentées. Le problème de la fatigue n'est pas uniquement concentré sur la soudure, le plein métal peut être la source de fissure. La contrainte admissible en fatigue pour un cas d'entaille plein métal varie fortement suivant qu'une pièce est en acier, en aluminium, en fonte GS ou encore au droit d'un bord libre mal découpé. Pour les châssis de bogies, la tenue en fatigue du métal de base a été déterminée à partir d'essais de fatigue en flexion 4 points.

Nos diagrammes de Goodman de référence pour les châssis de bogie ont été construits à partir de 


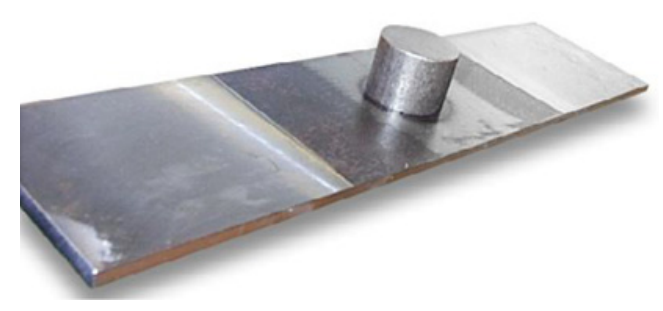

Figure 4. Exemple d'éprouvette de fatigue.

résultats d'essais de fatigue réalisés avec des éprouvettes détensionnées $[3,4]$.

Un châssis de bogie est dimensionné pour faire la vie de l'engin ( $\geq 30$ ans).

Sur la base de ces travaux, les vingt dernières années ont vu se développer les parachèvements de soudure, l'utilisation des aciers à hautes limite d'élasticité, montrant le potentiel encore disponible avec l'acier.

L'aluminium n'a pas par contre démontré ses capacités à résister aux sollicitations subies par cet organe, du en particulier à la probabilité d'apparition de fissures dont le risque de propagation était et reste non compatible avec les pas de maintenance.

A l'avenir, ce sont surtout les problématiques de maintenance qui guideront les évolutions : contrôle des serrages d'assemblages, fiabilisation des composants des bogies...

\section{Le freinage}

La solution retenue, et ceci depuis l'origine du chemin de fer, réside, presque exclusivement, dans l'application de pièces de frottement, appelées « sabots 》 puis «semelles 》 de frein, contre la surface de roulement en acier des roues.

Les matériaux de frottement utilisés se sont longtemps résumés à un seul : la fonte, si l'on excepte le bois utilisé à l'origine du chemin de fer et encore aujourd'hui sur certains métros. Depuis quelques années, des matériaux composites, puis frittés ont fait leur apparition [5].

Si ces dernières années, le ferroviaire a pu augmenter les vitesses de circulation, c'est grâce à la bonne maîtrise du freinage et donc de la distance d'arrêt de ses matériels. La principale difficulté du freinage est le contact acieracier puisque l'adhérence limitée entre la roue et le rail ne permet pas d'engendrer des efforts de retenue très importants. Les roues se bloquent et glissent sur le rail, rendant le freinage inefficace. La comparaison ci-après de deux environnements de freinage bien différents, l'un contact acier sur acier et l'autre caoutchouc sur asphalte illustre bien cette difficulté.

Un TGV de 485 tonnes roulant à $300 \mathrm{~km} / \mathrm{h}$ s'immobilisera en 3500 mètres en freinage d'urgence alors qu'un Boeing 747 de 360 tonnes, touchant la piste à une vitesse proche de $300 \mathrm{~km} / \mathrm{h}$, s'arrêtera en seulement 1700 mètres, sur une piste sèche. Un gros travail sur les différents composants, disques, semelles, garnitures, roues a permis l'obtention des résultats actuels.

La dernière décennie à surtout fait émerger la problématique du bruit au freinage et là encore, les matériaux ont permis d'améliorer la performance. Et dans

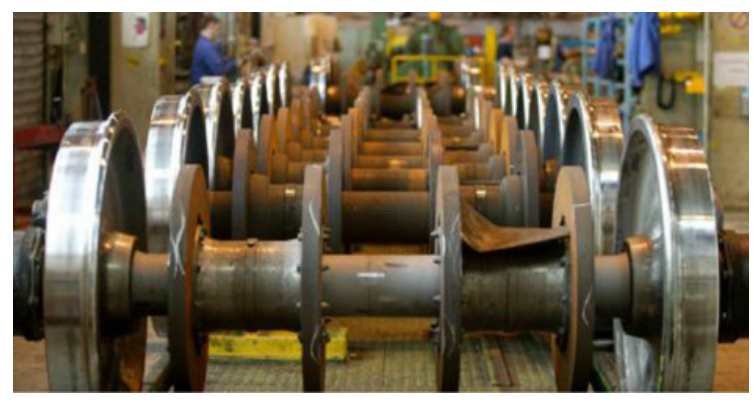

Figure 5. Essieux montés boités.

cette nouvelle étape, c'est la semelle fonte qui a presque disparu de tous les matériels voyageurs.

A l'avenir, ce sont les émissions au freinage qui seront un des sujets majeurs afin de respecter les contraintes environnementales.

\section{Les essieux}

Enfin, on ne peut pas parler d'optimisation de la performance sans évoquer les travaux menés sur les roues et les essieux axes.

Pour ces organes de sécurité par excellence que la SNCF s'est beaucoup impliquée dans la connaissance et la maîtrise des matériaux constitutifs des essieux ferroviaires, c'est probablement le prolongement des travaux historiques de l'ingénieur Wôlher (1819-1914) et du traumatisme résultant du déraillement sur la ligne Paris Versailles en 1842. Depuis cette époque, tous les travaux ont débouché sur un tryptique d'exigences en termes de qualité produit, de critères et de règles de conception robustes et en matière de contrôles et d'opérations de maintenance. Ces exigences justifiées ont souvent servis de base aux référentiels normatifs en vigueur.

L'avenir sera également tourné vers la maintenance au travers la recherche d'optimisation des opérations d'entretien notamment sur des roues toujours plus complexes intégrant des dispositifs de freinage (disques flasqués), des absorbeurs acoustiques... [6]

\section{L'ouverture sur l'Europe}

La cartographie des acteurs dans le ferroviaire a évolué. La SNCF est Détenteur de matériels, Entreprise Ferroviaire (EF) et Entité en Charge de la Maintenance (ECM) et cela parfois même pour d'autres EF [7]. Les missions sont bien différentiées ainsi que les responsabilités associées et cela résulte, entre autre de la normalisation Européenne. Les matériels qui circulent à travers les frontières devront être «interopérables».

Il faut à présent exprimer les CDC différemment pour tenir compte des obligations réglementaires européennes imposant l'abolition, entre les États membres, des obstacles à la libre circulation des marchandises, des personnes, des services et des capitaux. L'ouverture des marchés est à présent considérée comme un outil au service du développement du «marché commun». 


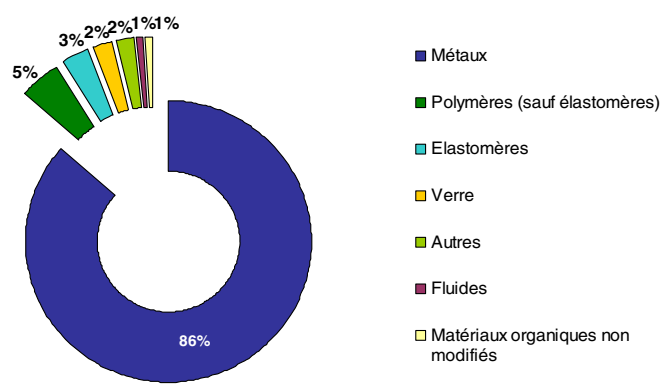

Figure 6. Répartition matière de Transilien.

Cette ouverture des CDC nous contraint à exprimer nos besoins d'une autre manière sans dégrader le niveau de Sécurité. De l'époque des études collaboratives et des développements communs avec les constructeurs et équipementiers on est à présent à l'ère des cahiers des charges fonctionnels auxquels et des notions de LCC (Life Cycle Cost). Cela sous entend qu'il nous faut pouvoir évaluer les propositions, savoir définir la maintenance et adapter et maîtriser les techniques de contrôle et de réparation. L'évolution des matériaux des matériels ferroviaires s'inscrit maintenant dans ces nouvelles dispositions. Il faut démontrer le caractère GAME (Globalement $\mathrm{Au}$ Moins Equivalent) d'une nouvelle proposition en intégrant son impact sur la maintenance jusqu'à sa fin de vie et son élimination et cela dans un contexte économique toujours plus difficile.

La dernière décennie a également vu émerger divers organismes chargés de garantir la sécurité des circulations ferroviaires dans le cadre de l'ouverture de la concurrence et de l'accès des réseaux à de multiples opérateurs. Des besoins apparaissent pour valider certaines règles de maintenance en s'appuyant non seulement sur le retour d'expérience comme le faisait jusqu'alors la SNCF seul exploitant du réseau, mais aussi sur des démonstrations qui amènent par exemple à s'intéresser à l'historique des chargements afin d'expliquer certaines défaillances et de mieux situer leur évolutions dans le temps afin de les confronter aux intervalles de maintenance. [8]

\section{LA PRISE EN COMPTE DU DÉVELOPPEMENT DURABLE}

Le renforcement de la réglementation sur l'environnement impose de maîtriser l'impact des matériels roulants [9]. La SNCF s'est engagée dès lors à développer des achats responsables [10]. Qu'en est-il pour les domaines qui nous intéressent?

Tout d'abord, il faut rappeler que malgré la progression des matériaux non métalliques en vue en particulier de l'allègement, les métaux représentent encore une part considérable dans la masse d'un train.

A titre d'exemple, le graphe ci-dessous représente la répartition en composition matière de l'automoteur Francilien :

Les nouveaux cahiers des charges demandent qu'au moins $85 \%$ des composants du matériel roulant soit recyclables et que celui-ci soit accompagné d'un manuel de démantèlement.

Progressivement sera imposée l'utilisation de matériaux recyclés, actuellement l'objectif est à $15 \%$.

Sans attendre, la SNCF à organisé, pour les matériels déjà existant et arrivant en fin de vie telles les voitures CORAIL, une procédure de démantèlement avec des soustraitants dument qualifiés.

Bien qu'à la frontière de notre domaine, il ne faut pas négliger les contraintes liées à l'utilisation des matériaux non métalliques, souvent compléments indispensables des structures et composants métalliques : lubrifiants, produits de nettoyage...

En application de la directive Européenne REACH, la SNCF est conduite à maîtriser, voire interdire l'utilisation de certains matériaux liées à l'utilisation des matériaux non métalliques et des produits chimiques... Elle s'intéresse entre autres à réduire l'impact des activités de maintenance et en particulier le nettoyage des pièces, pourtant indispensable, mais générateurs d'effluents qu'il faut impérativement traiter.

\section{CONCLUSION}

La SNCF a besoin de parfaitement connaître les matériaux constitutifs de ses matériels afin d'ajuster ses règles de maintenance en conséquences et ainsi garantir le haut niveau de Sécurité attendu par tous. Cela ne doit pas être un frein à la prospective et la recherche de nouveaux matériaux plus performants, plus résistants, plus économiques, plus écologiques. . .

De nouvelles préoccupations nous poussent à encore mieux connaître les matériaux utilisés et notamment leur comportement en service:

En parallèles aux études permettant de mieux décrire le comportement mécanique des matériaux face aux sollicitations du service, se développent des actions visant à satisfaire les contraintes environnementales. Ces actions sont les prémices du développement d'une économie circulaire. Il sera nécessaire pour cela de développer de nouvelles filières et d'industrialiser la réutilisation des matériaux

\section{Références}

[1] M. Bertrand : Métallisation des voitures à bogies à caisse en bois - RGCF 1938

[2] L. Guillaumat, F. Dau, F. Cocheteux, T. Chauvin: Reliability modeling of impacted composite materials for raiways: 16th Internatinal conference on composite materials au Japon, mars 2007

[3] M. Rimbaud, P. Lallet, JC Tourrade : L'allégement des châssis de bogies mécano-soudés des TGV - RGCF, nov 1993

[4] A. Leluan, M Rimbaud, JC Tourrade : Vers une codification $d u$ dimensionnement des bogies ferroviaires - RGCF, oct 1994

[5] J. Raison : Les attentes de la SNCF dans le domaine du frein - Le Rail, juin 2002 
[6] F. Cocheteux, W. d'Hardivilliers, S. Pommier, L. Gentot: Predictive maintenance for holed wheels determined by fracture mechanics: : $16^{\text {ème }}$ congrès des essieux montés à Cape town, mars 2010

[7] Règlement (UE) n ${ }^{\circ} 445 / 2011$ du 10 mai 2011

[8] N. Ranganathan*, N. Sedghi, D. Joly, T. D. Do, R. Leroy, F. Chalonand, P. Feraud: A Method for quantitative fatigue fracture surface analysis - The $4^{\text {th }}$ International Conference on Crack Paths (CP 2012)

[9] Rapport Responsabilité Sociétale de la SNCF qui acte l'engagement vis à vis du Développement Durable.

[10] M. Hosni, A. Kaddouri : L'intégration des critères de l'éco-mobilité dans les spécifications fonctionnelles des nouveaux matériels roulants - RGCF, mars 2009 